\title{
BMJ Open Efficacy of ultrasound therapy for the treatment of lateral elbow tendinopathy (the UCICLET Trial): study protocol for a three-arm, prospective, multicentre, randomised controlled trial
}

\author{
Ziyang Sun (D) , ${ }^{1,2}$ Shuai Chen (D) , ${ }^{1,2}$ Weixuan Liu (D) , ${ }^{1,2}$ Guixin Sun, ${ }^{3}$ \\ Junjian Liu (D) , Jian Wang, ${ }^{5}$ Wei Wang, ${ }^{1,2}$ Yuanyi Zheng (D) , ${ }^{6}$ Cunyi Fan (D) ${ }^{1,2}$
}

To cite: Sun Z, Chen S, Liu W, et al. Efficacy of ultrasound therapy for the treatment of lateral elbow tendinopathy (the UCICLET Trial): study protocol for a three-arm, prospective, multicentre, randomised controlled trial. BMJ Open 2022;12:e057266. doi:10.1136/ bmjopen-2021-057266

- Prepublication history and additional supplemental material for this paper are available online. To view these files, please visit the journal online (http://dx.doi.org/10.1136/ bmjopen-2021-057266)

ZS, SC and WL contributed equally.

ZS, SC and WL are joint first authors.

Received 11 September 2021 Accepted 03 December 2021

Check for updates

(c) Author(s) (or their employer(s)) 2022. Re-use permitted under CC BY-NC. No commercial re-use. See rights and permissions. Published by BMJ.

For numbered affiliations see end of article.

Correspondence to Professor Yuanyi Zheng; zhengyuanyi@sjtu.edu.cn and Professor Cunyi Fan; cyfan@sjtu.edu.cn

\section{ABSTRACT}

Introduction Lateral elbow tendinopathy (LET) is a highly prevalent disease among the middle-aged population, with no consensus on optimal management. Nonoperative treatment is generally accepted as the first-line intervention. Ultrasound (US) therapy has been reported to be beneficial for various orthopaedic diseases, including tendinopathy. The purpose of this study is to investigate the efficacy of US for LET treatment.

Methods and analysis This protocol entails a three-arm, prospective, multicentre, randomised controlled trial. Seventy-two eligible participants with clinically confirmed LET will be assigned to either (1) US, (2) corticosteroid injections or (3) control group. All participants will receive exercise-based therapy as a fundamental intervention. The primary outcome is Patient-rated Tennis Elbow Evaluation. The secondary outcomes include Visual Analogue Scale for pain, shortened version of the Disabilities of the Arm, Shoulder and Hand for upper limb disability, pain free/ maximum grip strength, Work Limitations Questionnaire-25 for functional limitations at work, EuroQol-5D for general health, Hospital Anxiety and Depression Scale for mental status, Global Rating of Change for treatment success and recurrence rate, and Mahomed Scale for the participant's satisfaction. Adverse events will be recorded. Intention-totreat analyses will be used.

Ethics and dissemination Ethics committees of all clinical centres have approved this study. The leading centre is Shanghai Sixth People's Hospital, whose approval number is 2021-153. New versions with appropriate amendments will be submitted to the committee for further approval. Final results will be published in peerreviewed journals and presented at local, national and international conferences.

Trial registration number ChiCTR2100050547.

\section{INTRODUCTION}

First described by Runge, ${ }^{1}$ lateral elbow tendinopathy (LET), also widely known as tennis elbow, has an estimated prevalence of $1 \%-3 \%$ in the general population, and peaks at the fourth and fifth decades of life, with

\section{Strengths and limitations of this study}

Exercise-based therapy as a fundamental intervention for all participants.

- The first randomised controlled trial (RCT) to compare the efficacy between ultrasound therapy and corticosteroid injections in lateral elbow tendinopathy treatment.

Multicentre RCT with blinded outcome assessor and statistician.

- Use of several patient-reported outcome measures as well as objective parameters.

- Participants and treating surgeons not blinded.

an equal gender distribution. ${ }^{2}$ LET causes a great burden on the social economy, with an annual sickness absence rate as high as $5 \%$ in working-aged adults. ${ }^{3}$ Though previously considered as 'tendinitis', histological analysis suggests a degenerative rather than an inflammatory process in LET, which is now commonly converted to be considered as a 'tendinosis'. ${ }^{4}$ A LET diagnosis is usually straightforward, with clear clinical signs and symptoms. The patient most often presents with pain at or around the bony surface of the upper half of the lateral epicondyle and is likely to have a history of strenuous overuse relating to particular repetitive actions in the affected upper limb. ${ }^{56}$

Though LET usually is a self-limiting condition, complaints may last up to 2 years or longer, ${ }^{7}$ therefore, it has great clinical value to find a better and faster recovery process. General principles of LET treatment should be orientated towards pain relief, movement restoration, grip strength and endurance improvement, return to normal function and life quality, and control of further clinical deterioration. ${ }^{8}$ Treatments can be divided 
into operative and non-operative therapies. Invasive treatments commonly include open, arthroscopic and percutaneous release of the common extensor origin. ${ }^{9}$ Among these, ultrasonic percutaneous tenotomy, a recently developed method, appealing to many researchers for its good durability of pain relief and functional recovery, ${ }^{10}$ has satisfactory long-term (90 months) outcomes reported by Ang et al. ${ }^{11}$ However, surgery is usually considered for patients with persistent pain and disability after a course of well-performed conservative therapy, with a proportion as low as $3 \%$ in the whole LET population; ${ }^{2}$ therefore, nonoperative treatment is suggested as first-line treatment. ${ }^{12}$ Generally, non-surgical methods include injections (like corticosteroid, platelet-rich plasma, autologous blood, sodium hyaluronate, and so on), physiotherapy, extracorporeal shock-wave therapy (ESWT), ultrasound (US), topical glyceryl trinitrate, or oral naproxen, and so on. ${ }^{13} 14$

So far, despite the wide range of treatments, there is no successful and universally accepted regimen. In a cross-sectional survey of UK practice in managing LET, $81 \%$ of experts recommended exercise-based therapy (EBT) as the first choice of intervention. ${ }^{15}$ EBT was also supported by high-quality clinical trials ${ }^{16-18}$ and systematic reviews, ${ }^{19} 20$ regarded as the most cost-effective treatment for LET. ${ }^{21}$ The survey also showed that, as the mainstream treatment for a long time, corticosteroid injection (CI) was still the most recommended intervention second to $\mathrm{EBT},{ }^{15}$ due to its quick pain relief and physical functional improvement, though the recurrence rate may be high and prognosis may be worsened in the long term. ${ }^{16-18}$ In addition, systematic reviews have shown that the effects of other conservative treatments like autologous blood or hyaluronate injection, ${ }^{22}$ platelet-rich plasma injection, ${ }^{23}$ ESWT $^{24}$ and acupuncture ${ }^{25}$ remain controversial or provide little to no benefit.

US is widely used for imaging purposes and regarded as an adjunct to physiotherapy. US can reduce muscle spasms and pain, and facilitate tissue repair by increasing local blood flow and stimulating inflammatory mediators. ${ }^{26}$ US has been widely reported to be treatment beneficial in fracture non-unions, ${ }^{27}{ }^{28}$ osteoarthritis, ${ }^{29}{ }^{30}$ chronic muscle pain,${ }^{31} 32$ soft tissue injury, ${ }^{33}$ and so on. As for tendinopathy, US is also a potential non-invasive treatment modality for frozen shoulder, ${ }^{3435}$ rotator cuff, ${ }^{36}$ achilles ${ }^{3738}$ and patellar ${ }^{39}$ tendinopathy. Some studies have reported the efficacy of US in LET treatment, but with low grade of study design and data, ${ }^{40}$ and most of them focused on the comparison between US and ESWT. ${ }^{41-45}$ Both Yalvaç $\mathrm{B}^{43}$ and Özmen $\mathrm{T}^{41}$ have shown significant improvements in pain, upper limb function, strength and quality of life from baseline after treatment with US. However, they did not have a control group, which would make it unclear whether the efficacy comes from US itself or the passing time, as LET is a self-limiting disease. Therefore, the role of US in LET treatment still needs to be further explored by high-quality studies. Additionally, to our best knowledge, no study has compared the efficacy between US and CI in LET treatment yet.
Therefore, the purpose of the current three-arm, prospective, randomised, multicentre trial is to investigate the efficacy of US in treatment for LET, that is, US versus $\mathrm{CI}$ versus control, with a fundamental intervention of EBT, on clinical and functional outcomes, including Patient-rated Tennis Elbow Evaluation (PRTEE). In view of recent literatures, CI should be discouraged in LET; ${ }^{22} 46$ however, it is still common in clinics due to the ability to satisfy patients' need for quick pain relief. ${ }^{15}$ Thus, a change in the paradigm of LET treatment is necessary. This change will come about through proposed evidence-based treatment guidelines. There have been some ongoing clinical trials on LET treatment in recent years, ${ }^{47-49}$ and our prospective randomised controlled trial (RCT) proposes to complement and add to this relevant and much needed scientific effort.

\section{METHODS}

\section{Study design}

The design of this study is a three-arm, prospective, multicentre RCT that will enrol participants with a diagnosis of chronic symptomatic LET from four municipal tertiary hospitals (Shanghai Sixth People's Hospital, Shanghai East Hospital, Shanghai Tenth People's Hospital, and Pudong New Area People's Hospital of Shanghai). This manuscript is written according to the Standard Protocol Items: Recommendations for Interventional Trials (SPIRIT) guidelines. ${ }^{50}$ The name of this trial is Ultrasound versus Corticosteroid Injections versus Control in Lateral Elbow Tendinopathy (UCICLET).

\section{Participant and public involvement}

This study was done without participant involvement. Participants were not invited to comment on the design, consulted to choose patient-relevant outcomes, invited to contribute to the writing or editing of this manuscript for readability or accuracy. The final results and related publications will be disseminated to the public via mass media. Participants as a whole will be acknowledged at the end of our publications and presentations.

\section{Participant recruitment}

Figure 1 shows the participant flow chart throughout this study. Participants will be recruited over a period of 5 months, from the intake clinics of four principals of each subcentre. Additionally, participants will also be recruited through other physicians and healthcare professionals. Those interested will contact the research assistant who will provide further information about the study objectives and procedures and will perform an initial eligibility screening interview by telephone.

\section{Medical evaluation and enrolment procedure}

Participants potentially eligible will be invited to attend a medical examination to confirm the LET diagnosis and assess eligibility to participate in the research project. 


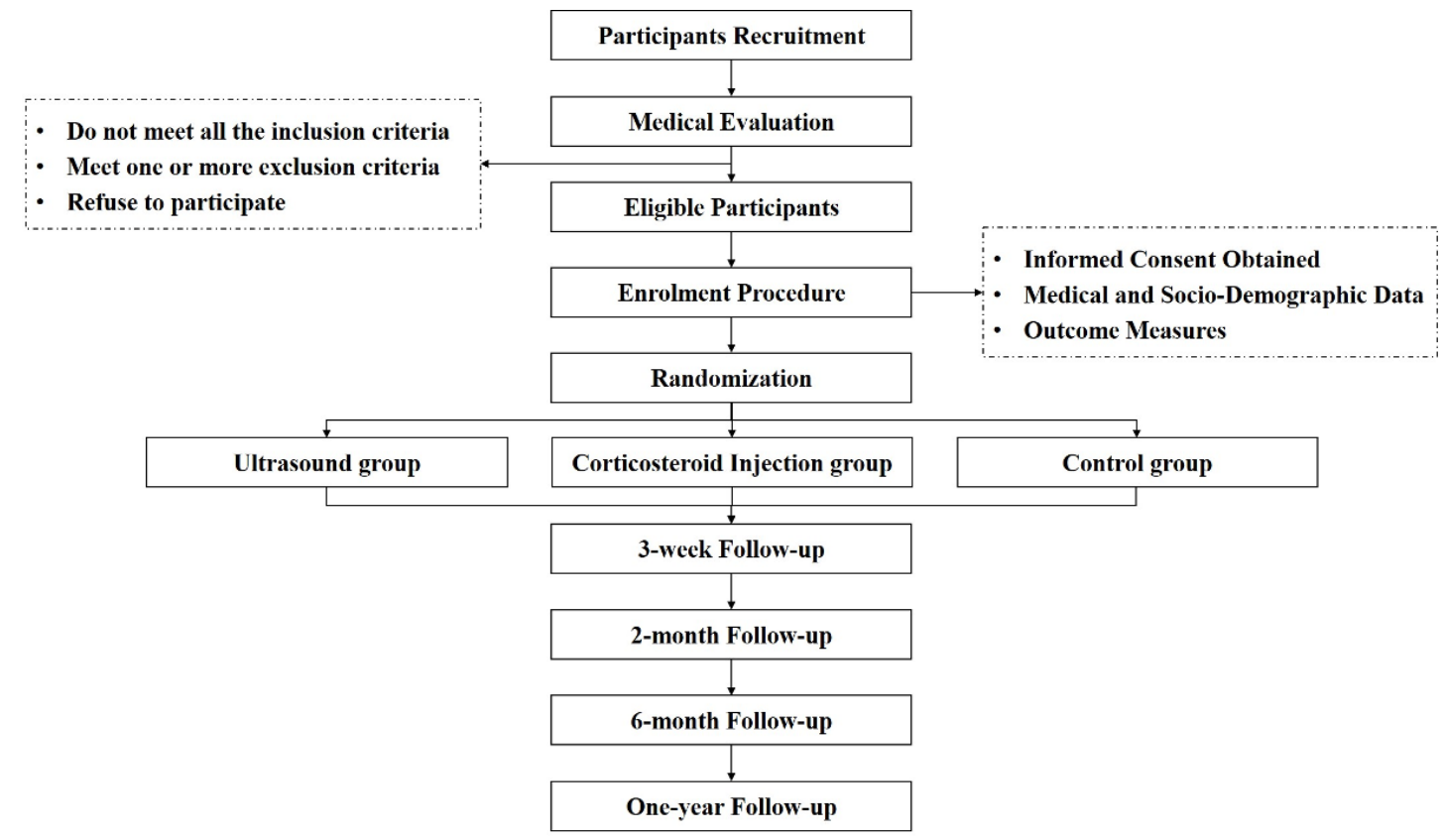

Figure 1 Participant flow chart.

\section{Inclusion criteria}

- Age $\geq 18$ years old;

- Unilateral lateral elbow pain longer than 6 weeks duration;

- Pain severity over the lateral humeral epicondyle greater than $30 \mathrm{~mm}$ on a $100 \mathrm{~mm}$ Visual Analogue Scale (VAS), provoked by at least two of the following: gripping, palpation, resisted wrist or middle finger extension, or stretching of forearm extensor muscles with reduced pain-free grip; ${ }^{16} 49$

- Able to read and write in simplified Chinese (Mainland), understand and complete the questionnaire, and provide informed consent.

\section{Exclusion criteria}

- Concomitant musculoskeletal pain conditions reported by participants to be their predominant complaint within the past 6 months;

- History of symptoms suggesting radicular, neurological, inflammatory or systemic arthritic conditions;

- Treatment by physiotherapy, electrophysical therapy, or injection within the past 6 months, or previous tennis elbow surgery;

- Contraindications to US, including dermatological conditions, abnormal sensation in the affected arm, indwelling electrical pumps/pacemakers, epilepsy, pregnancy or breastfeeding, and so on;

- Contraindications to CI, including hypertension, gastrointestinal ulcers, diabetes, mental illness, and so on.

Following the medical evaluation, a research assistant will meet with the eligible participants and obtain written informed consent. Demographic variables will be reported before treatment (baseline) of all participants regarding age, sex, body mass index, affected elbow, dominant arm, lifestyle (smoking and drinking) and previous medical history. Participants will also be asked relevant questions about the duration of symptoms and previous treatments (rehabilitation exercises, injections or others). Others like occupation, employment characteristics (full-time or part-time work, manual or nonmanual labour), employment status (whether on sickness absence), professional activity characteristics (repetitive movements for $>4$ hours/day; wrist flexion or extension for $>2$ hours/day; use of computer keyboard/mouse or vibrating instruments (how many hours/day)), and sports activities (how many hours/week, activity type, team or individual sports) ${ }^{51}$ will also be collected.

\section{Randomisation and blinding}

Participants will be randomised into three intervention groups (either US or CI or control arm) in a ratio of 1:1:1, using a computer-generated randomised sequence with varying unknown block sizes (either three or six) for all study centres, without stratification. A research assistant with no involvement in the clinical care or outcome evaluations will prepare sequentially numbered, opaque, sealed envelopes according to the randomisation lists, with security in place to ensure allocation data cannot be accessed or influenced by any person. At the appropriate time, this assistant will open the envelope and assure coordination of the therapeutic interventions.

The outcome assessor and statistician will be blinded to group allocation and not involved in treatment procedures.

\section{Intervention}

At the beginning, all participants will receive standardised education and advice on adjusting activity patterns and managing pain, which will be distributed 
in the form of printed brochures and orally assessed on their understanding of the content. Participants will be told that absolute rest of the arm will not be advocated, and activities that do not cause elbow pain should be encouraged. The primary physical impairment in LET, which occurs in the muscle system, is best characterised as a deconditioning response of the forearm muscles to the pain. Therefore, all participants will receive the internationally best recommended fundamental intervention, EBT programme, for the forearm muscles. ${ }^{15}$ The EBT in this study will follow a standard protocol that has been adopted and used by several high-quality RCTs, ${ }^{16} 185253$ mainly for addressing motor impairments, relieving pain and stimulating tendon remodelling. Thirty minutes per day, including basic tasks (pain-free (1) gripping and (2) extension exercise) and appendage tasks ((3) flexion, (4) supination and pronation, and (5) radial and ulnar deviation exercise). Various kinds of resistance and loads can be used, like free weights, rubber bands, manual resistance, isokinetic dynamometry or isometric contractions. (6) It is essential that all exercises performed for the upper limb be done with sound alignment of the spine, trunk and proximal arm.

1. Pain-free gripping exercise with exercise putty, which allows practice of various gripping actions.

2. Forearm extensor muscle exercise using a freestanding dumbbell. Note that the forearm is fully stabilised by the bench and upper body in sound postural alignment. Duration per repetition lasts about 6-10s.

3. Dumbbell weight exercise for the forearm flexor muscle with $6-10$ s per repetition. The postural is the same as 2).

4. Exercises for forearm supinator and pronator muscles using an imbalanced adjustable dumbbell weight with $6-10$ s per repetition, from end range of supination to pronation with the participant maintaining full active control of the weight. The elbow bent to $90^{\circ}$ with the arm stabilising beside the trunk. Progressions in load imposed on the muscles can be achieved by increasing the weight or the distance between weight and hand.

5. Radial and ulnar deviation exercises are performed with similar equipment and guidelines in 4 .

6. Education on recognition and correction of the poor posture from the pelvis to neck. Once the spine and trunk are aligned more optimally, the upper limb position should be addressed.

Participants in the US group will receive continuous mode US (Shanghai, China) at a frequency of $1 \mathrm{MHz}$ and intensity of $1.0 \mathrm{~W} / \mathrm{cm}^{2}$ for $10 \mathrm{~min}, 5$ days per week for 3 weeks on the maximum pain region of the lateral elbow.

Participants allocated to the CI group will receive a single local infiltration of $1 \mathrm{~mL}$ triamcinolone acetonide $(10 \mathrm{mg} / \mathrm{mL})$ and $1 \mathrm{~mL}$ lidocaine $1 \%$. Local CI was administered to the most painful area on pressure around the lateral epicondyle. Participants will be advised to wait for 20 min following injection and inform their doctor if there is any suggestion of infection or other adverse events. All adverse reactions will be managed by a committee chaired by the chief investigator. Rest from all strenuous activity for 1-2 weeks following injection will be strongly recommended, followed by a gradual return to normal activities. Participants will be instructed to avoid an aggressive return to activities even if substantial relief is obtained to minimise the potential recurrence of their symptoms.

Participants randomised to the control group will neither receive US therapy nor CI. They will only receive the fundamental intervention, EBT programme.

We discourage additional treatments to that assigned (that is, not per protocol) during the intervention period, but we allow the use of simple analgesics as needed. Participants will report all not-per-protocol treatments, such as drugs, in a diary.

\section{Data management}

Data will be collected during the participants' visits to the hospital at baseline, 3 weeks, 2 months and 6 months, and 1 year after random assignment (table 1 ). In order to maximise participant compliance in follow-up completion, reminder emails and a telephone call by the research assistant will be programmed. Registered participants will be withdrawn from the study if: (1) Participant withdraws his/her consent, and (2) Exclusion criteria is discovered after registration. The reason and date of discontinuation will be recorded. Consent to use the data already collected prior to a participant's withdrawal will be included in the consent form.

\section{Outcome measures}

\section{Primary outcome}

The primary outcome measure will be the difference in PRTEE. The PRTEE, formerly known as the PatientRated Forearm Evaluation Questionnaire, is a wellvalidated composite scale measuring pain (five items, with $0=$ no pain and $10=$ worst imaginable) and physical function (six items for specific activities and four items for usual activities, with $0=$ no difficulty and $10=$ unable to do), ${ }^{54}$ ranging from 0 to 100 points, with higher scores representing worse possible pain and more loss of function. The pain (intraclass correlation coefficients, $\mathrm{ICC}=0.89$ ), physical function $(\mathrm{ICC}=0.83$ ) and the total $(\mathrm{ICC}=0.89)$ scores all demonstrate excellent reliability. ${ }^{55}$ A variation of $11 / 100$ points or $37 \%$ of baseline scores are reported for clinical significance defined as 'much better' or 'completely recovered'. ${ }^{56}$ We use a validated Hong Kong Chinese version ${ }^{57}$ of the PRTEE translated into simplified Chinese (Mainland) because the culture and language are the same.

\section{Secondary outcome}

Secondary outcome measures will be the differences in VAS $^{58}$ for pain, shortened version of the Disabilities of the Arm, Shoulder and Hand (Quick-DASH) ${ }^{59}$ for upper limb disability, pain-free/maximum grip strength, Work Limitations Questionnaire-25 (WLQ-25) ${ }^{60}$ for functional limitations at work, EuroQol-5D (EQ-5D ${ }^{61}$ for life quality and health status, the Hospital Anxiety and Depression 
Table 1 Study evaluation procedures and timeline

\begin{tabular}{|c|c|c|c|c|c|c|}
\hline Study procedure & $\begin{array}{l}\text { Medical } \\
\text { evaluation }\end{array}$ & $\begin{array}{l}\text { Enrolment } \\
\text { visit }\end{array}$ & 3 weeks & 2 months & 6 months & 1 year \\
\hline Determine eligibility & $\sqrt{ }$ & $\sqrt{ }$ & & & & \\
\hline Obtain medical and demographic data & & $\sqrt{ }$ & & & & \\
\hline Give instructions for pain medication diary & & $\sqrt{ }$ & & & & \\
\hline Visual Analogue Scale for pain & & $\sqrt{ }$ & $\sqrt{ }$ & $\sqrt{ }$ & $\sqrt{ }$ & $\sqrt{ }$ \\
\hline $\begin{array}{l}\text { Shortened version of the Disabilities of the Arm, } \\
\text { Shoulder and Hand Questionnaire }\end{array}$ & & $\sqrt{ }$ & $\sqrt{ }$ & $\sqrt{ }$ & $\sqrt{ }$ & $\sqrt{ }$ \\
\hline Pain-free/maximum grip strength & & $\sqrt{ }$ & $\sqrt{ }$ & $\sqrt{ }$ & $\sqrt{ }$ & $\sqrt{ }$ \\
\hline Treatment success rate & & & $\sqrt{ }$ & $\sqrt{ }$ & $\sqrt{ }$ & $\sqrt{ }$ \\
\hline Treatment recurrence rate & & & & $\sqrt{ }$ & $\sqrt{ }$ & $\sqrt{ }$ \\
\hline Participants' satisfaction & & & $\sqrt{ }$ & $\sqrt{ }$ & $\sqrt{ }$ & $\sqrt{ }$ \\
\hline
\end{tabular}

Scale (HADS) ${ }^{62}$ for anxiety and depression status, Global Rating of Change (GROC) for treatment success and recurrence rate, and Mahomed Scale ${ }^{63}$ for participants' satisfaction.

\section{Pain}

VAS will be used for pain evaluation, which consists of a $100 \mathrm{~mm}$ horizontal numbered line anchored at one end $(0)$ with the words 'no pain' and at the other end (100) with the words 'worst pain imaginable'. The score is determined by the distance between the left end of the line and the participant's mark in $\mathrm{mm} .{ }^{58}$ VAS is considered to be the most sensitive of all pain scoring scales and has been specifically validated in the LET population with high reliability $(\mathrm{r}=0.89)$ and a moderate correlation with pain-free grip strength $(\mathrm{r}=0.47) .{ }^{64}$ Participants will be asked to score their pain on this line during rest (at time of measure), provocation and maximum grip strength. The provocation test is conducted on the outpatient clinic by resisted wrist dorsiflexion during full elbow extension. Clinically relevant improvement will be defined when a $50 \%$ decrease in VAS is observed before and after the treatment. ${ }^{65}$ The consumption of rescue medication taken by each patient will be also recorded at each follow-up visit.

\section{Upper limb disability}

The well-validated simplified Chinese (Mainland) version of Quick-DASH ${ }^{66}$ will be used for elbow function evaluation, consisting of eleven questions scored on a 5-point scale similar to DASH. ${ }^{59}$ Total and individual module scores will be calculated out of 100 , with a higher score indicating a worse status. A minimal clinically important difference of 15.91 points has been reported. ${ }^{67}$

\section{Grip strength}

Pain-free/maximum grip strength will be measured using a dynamometer (CAMRY, City of Industry, California, USA). The participants will be asked to take a shoulder-width stance and allow their arms to hang loose, holding their arm adducted along the body and the elbow in full extension. The pain-free grip strength will be measured, followed by the maximum grip strength, and the affected side will be measured first and then the unaffected side. The measurement readings will be not revealed to the subjects until the completion of the test. The pain-free grip strength will be measured up to the point when the subject slowly squeezes the dynamometer until the occurrence of pain. The maximum grip strength will be measured at the maximum grip level. The mean of three consecutive trials, separated by a $20 \mathrm{~s}$ pause, will be calculated. Results will be presented as a ratio of values of the symptomatic side/asymptomatic side $\times 100 .^{68}$

\section{Functional limitations at work}

In order to gather the information that is complementary to the pain and disability scales, functional limitations at work will be measured with WLQ-25. It contains 25 items arranged under four subscales addressing four dimensions of job demands: time demands, physical demands, mental/interpersonal demands and output demands. ${ }^{60}$ A five-level ordinal response scale ranging from 0 (all of the time) to 4 (none of the time) with an additional sixth option (does not apply to my job) is used. The total scores range from 0 to 100 points, and a 13-point (out of 100) improvement for the summed score is established for clinically important differences. ${ }^{69}$ 


\section{Life quality and health status}

The EQ-5D is a widely validated generic health-related quality of life (HRQol) measure known for its simplicity. ${ }^{61}$ It contains a five-dimension descriptive system (mobility, self-care, usual activities, pain/discomfort and anxiety/ depression) and a VAS, ranging from 0 to 1 , in which one represents perfect health. All the dimensions are grouped into three levels (no problem, some problem and extreme problem). We used a validated Chinese version $^{70}$ of the EQ-5D, which has been recommended by China Guidelines for Pharmacoeconomic Evaluations 2011 for a measure for HRQol and health utility. ${ }^{71}$

\section{Anxiety and depression status}

HADS will be used to identify and quantify two of the most common psychological disorders, anxiety and depression. ${ }^{62}$ There is evidence of increased levels of anxiety and depression in people with LET ${ }^{72}$ HADS is a 14-item scale independent of somatic symptoms, which consists of two 7-item subscales measuring depression and anxiety, respectively. A 4-point scale (from 0 representing the absence of symptoms to 3 representing the maximum symptomatology) is used. The total scores for each subscale range from 0 to 21 points, with higher scores indicating higher levels of disorder. HADS has two cut-offs for categorisation: 0-7, 'non-case'; 8-10, 'possible or doubtful case'; 11-21, 'probable or definite case'.

\section{Treatment success and recurrence rate}

Participants' treatment impressions of change regarding their condition will be recorded on a 6-point Likert Scale (from 'completely recovered', 'much improved', 'somewhat improved', 'same', 'worse' to 'much worse'). Success rates will be calculated by dichotomising responses. Participants who report their overall condition as 'completely recovered' or 'much improved' since the beginning of the study will be counted as successes, while other responses will be counted as failures. ${ }^{16} 18$ Recurrence will primarily be defined as occurring when a participant rates a success at 3 weeks and a failure at 2 months or 6 months or 1 year on the GROC. ${ }^{16} 18$

Additional treatments will also be recorded after the failure of management in this study (that is, not per protocol), if any, including subsequent interventions and even surgery.

\section{Participants' satisfaction}

Similarly, participants' level of satisfaction on the evolution of their condition will be determined on a validated 4-point Likert Scale ranging from 'very satisfied', 'somewhat satisfied', 'somewhat dissatisfied' to 'very dissatisfied'. ${ }^{74}$

\section{Adverse events}

All adverse events, defined as any negative or unwanted reactions to intervention, will be recorded through the symptoms reported by the participants, and observations by the researcher at every visit. US treatment may cause mild local swelling, spot-like bleeding, ecchymosis, enhanced local pain response, and local hyperesthesia or decrease. CI-related adverse events are divided into acute and long-term ones. Acute events include dizziness, skin flushing, local bleeding, and some may even develop rarer physical reactions, such as arrhythmias. Therefore, all participants must take at least $20 \mathrm{~min}$ in the outpatient room to observe and even manage any acute adverse reactions following the injection. Long-term events may cause skin pigmentation, local calcification and infection.

\section{Sample size calculation}

Sample size and power calculation are based on the primary outcome of the PRTEE Score. All sample size calculations assume two-sided analysis with a power of $90 \%(1-\beta=0.90)$ at a significant level of $\alpha=0.05$. A SD of 5.1 points on the PRTEE Score will be used based on the previous trial. ${ }^{75}$ To detect a minimum clinically significant difference of 11.0 points $^{56}$ (superiority margin) between the US and control groups (assuming a true difference of 15.6 points), ${ }^{43} 75$ a total of 22 participants in each group is required. Allowing for an up to $10 \%$ dropout rate, we aim to enrol at least 24 participants in each group to complete the study.

\section{Analysis plan}

Baseline characteristics will be summarised for the three treatment groups using appropriate descriptive statistics. Both primary and secondary analyses will be conducted blinded to treatment allocation and analysed on the intention-to-treat ${ }^{76}$ approach with all randomised participants retaining their original randomised group. Multiple imputation by chained equations will be used to address missing data caused by loss to follow-up and nonresponses if these missing data are judged to be random.

The primary comparisons for the PRTEE Scores will be made using linear regression. In secondary analyses, a repeated measures mixed model ${ }^{77}$ will also be used to examine the associations between treatments and repeated outcome measures, with terms of treatment, time, trial centre and corresponding baseline values as covariates (age, gender, body mass index, and so on). Linear regression will be used for numerical outcomes and logistic/ordinal regression for any categorical outcomes.

\section{Quality assurance/monitoring/management}

To standardise staff training and study procedures like participant recruitment, outcome measurement, data import, security, management and analysis, a Manual of Operations and Procedures and a case report form will be developed as per protocol, which also include the monitoring plans to assure participant protection and data integrity, thus facilitating consistency in protocol implementation and data collection. The investigators, physicians, research assistants, outcome assessors and statisticians are different people and should receive Good Clinical Practice training. A trained project manager will 
visit each centre for monitoring to ensure data quality and compliance with the trial protocol.

All data obtained will be kept strictly confidential and stored electronically in a database with secured access. Encryption will be used for data transfer, with removal of any information that could identify individuals. De-identification of data will only be permitted at the final of this trial for analysis.

\section{Study duration}

Recruitment will begin in November 2021, and 1-year follow-up for all participants is anticipated to be completed by March 2023. See table 1 for time points and recruitment progress.

\section{Ethics and dissemination}

The trial has been approved by all four medical ethics committees: Ethics Committee of Shanghai Sixth People's Hospital (the leading clinical centre, approval No. 2021-153), Ethics Committee of Shanghai East Hospital (EC.D(BG).016.03.1-2021-096), Ethics Committee of Shanghai Tenth People's Hospital (SHSYIEC-4.1/21-193/01) and Ethics Committee of Pudong New Area People's Hospital (IRBY2021-005). The potential risks of this clinical trial are considered to be minimal and are addressed in the protocol and consent forms. A written consent (online supplemental file 1) will be obtained by clinical practitioners from each participant. The trial was registered on the www.chictr.org website (registration number ChiCTR2100050547). Data will be published in peer-reviewed journals and presented at conferences, both nationally and internationally.

\section{Limitation}

This study will have one limitation. Participants and treating surgeons are inevitably not blinded, which may produce bias. However, we will strictly control the outcome assessors and statisticians to be blinded to group allocation and not involved in treatment procedures to reduce the bias.

\section{Author affiliations}

${ }^{1}$ Department of Orthopedics, Shanghai Jiao Tong University Affiliated Sixth People's Hospital, Shanghai, China

${ }^{2}$ Shanghai Engineering Research Center for Orthopaedic Material Innovation and Tissue Regeneration, Shanghai, China

${ }^{3}$ Department of Orthopaedics, Shanghai East Hospital, Tongji University School of Medicine, Shanghai, China

${ }^{4}$ Department of Orthopedics, Shanghai Tenth People's Hospital, School of Medicine, Tongji University, Shanghai, China

${ }^{5}$ Department of Orthopaedics, Pudong New Area People's Hospital, Shanghai, China ${ }^{6}$ Department of Ultrasound in Medicine, Shanghai Jiao Tong University Affiliated Sixth People's Hospital, Shanghai, China

Acknowledgements The authors thank the Base for Interdisciplinary Innovative Talent Training, Shanghai Jiao Tong University and Youth Science and Technology Innovation Studio of Shanghai Jiao Tong University School of Medicine for their support.

Contributors ZS and SC are the primary investigators. ZS, SC, WL, YZ, CF participated in the development of the study design. ZS, SC, WL, GS, JL, JW, WW, YZ and CF participated in the study conduct. ZS, SC and WL drafted the manuscript under CF's supervision. CF contributed to applying for and gaining funding. All authors contributed to the content and critical revision and approved the final draft of the manuscript.

Funding This study will be supported by General Project of National Natural Science Foundation of China (8217090787); Shanghai Engineering Technology Research Center and Professional Technology Service Platform project of 2020 'Science and Technology Innovation Action Plan' of Shanghai (20DZ2254100); Municipal Hospital Clinical Skills and Innovation Capacity of Three-year Action Plan Program of Shanghai Shenkang Hospital Development Center (SHDC2020CR2039B, SHDC2020CR6019-002); Biomedical Technology Support Special Project of Shanghai 'Science and Technology Innovation Action Plan' (20S31900300, 21S31902300); Clinical Research Center (CRC) of Shanghai University of Medicine and Health Sciences (20MC2020001).

Competing interests None declared.

Patient consent for publication Consent obtained directly from patient(s).

Provenance and peer review Not commissioned; externally peer reviewed.

Supplemental material This content has been supplied by the author(s). It has not been vetted by BMJ Publishing Group Limited (BMJ) and may not have been peer-reviewed. Any opinions or recommendations discussed are solely those of the author(s) and are not endorsed by BMJ. BMJ disclaims all liability and responsibility arising from any reliance placed on the content. Where the content includes any translated material, BMJ does not warrant the accuracy and reliability of the translations (including but not limited to local regulations, clinical guidelines, terminology, drug names and drug dosages), and is not responsible for any error and/or omissions arising from translation and adaptation or otherwise.

Open access This is an open access article distributed in accordance with the Creative Commons Attribution Non Commercial (CC BY-NC 4.0) license, which permits others to distribute, remix, adapt, build upon this work non-commercially, and license their derivative works on different terms, provided the original work is properly cited, appropriate credit is given, any changes made indicated, and the use is non-commercial. See: http://creativecommons.org/licenses/by-nc/4.0/.

\section{ORCID iDs}

Ziyang Sun http://orcid.org/0000-0002-8673-9521

Shuai Chen http://orcid.org/0000-0003-3724-0174

Weixuan Liu http://orcid.org/0000-0002-2010-3010

Junjian Liu http://orcid.org/0000-0002-3052-3799

Yuanyi Zheng http://orcid.org/0000-0002-1328-0641

Cunyi Fan http://orcid.org/0000-0002-7854-5233

\section{REFERENCES}

1 Knobloch K, Gohritz A. Dr Runge: a German pioneer in sclerosing therapy in epicondylitis in 1873. Br J Sports Med 2010. doi:10.1136/ bjsm.2008.051326. [Epub ahead of print: 16 Nov 2010].

2 Sanders TL, Maradit Kremers H, Bryan AJ, et al. The epidemiology and health care burden of tennis elbow: a population-based study. Am J Sports Med 2015;43:1066-71.

3 Walker-Bone K, Palmer KT, Reading I, et al. Occupation and epicondylitis: a population-based study. Rheumatology 2012;51:305-10.

4 Khan KM, Cook JL, Kannus P, et al. Time to abandon the "tendinitis" myth. BMJ 2002;324:626-7.

5 Haahr JP, Andersen JH. Physical and psychosocial risk factors for lateral epicondylitis: a population based case-referent study. Occup Environ Med 2003;60:322-9.

6 Herquelot E, Guéguen A, Roquelaure Y, et al. Work-Related risk factors for incidence of lateral epicondylitis in a large working population. Scand J Work Environ Health 2013;39:578-88.

7 Hudak PL, Cole DC, Haines AT. Understanding prognosis to improve rehabilitation: the example of lateral elbow pain. Arch Phys Med Rehabil 1996;77:586-93.

8 Ahmad Z, Siddiqui N, Malik SS, et al. Lateral epicondylitis: a review of pathology and management. Bone Joint J 2013;95-B:1158-64.

9 Pierce TP, Issa K, Gilbert BT, et al. A systematic review of tennis elbow surgery: open versus arthroscopic versus percutaneous release of the common extensor origin. Arthroscopy 2017;33:1260-8.

10 Vajapey S, Ghenbot S, Baria MR, et al. Utility of percutaneous ultrasonic tenotomy for Tendinopathies: a systematic review. Sports Health 2021;13:258-64.

11 Ang BFH, Mohan PC, Png MA, et al. Ultrasonic percutaneous tenotomy for recalcitrant lateral elbow tendinopathy: clinical 
and sonographic results at 90 months. Am J Sports Med 2021;49:1854-60.

12 Vaquero-Picado A, Barco R, Antuña SA. Lateral epicondylitis of the elbow. EFORT Open Rev 2016:1:391-7.

13 Lian J, Mohamadi A, Chan JJ, et al. Comparative efficacy and safety of nonsurgical treatment options for Enthesopathy of the extensor Carpi Radialis brevis: a systematic review and metaanalysis of randomized placebo-controlled trials. Am J Sports Med 2019;47:3019-29.

14 Sayegh ET, Strauch RJ. Does nonsurgical treatment improve longitudinal outcomes of lateral epicondylitis over no treatment? A meta-analysis. Clin Orthop Relat Res 2015;473:1093-107.

15 Bateman M, Titchener AG, Clark DI, et al. Management of tennis elbow: a survey of UK clinical practice. Shoulder Elbow 2019;11:233-8.

16 Coombes BK, Bisset L, Brooks P, et al. Effect of corticosteroid injection, physiotherapy, or both on clinical outcomes in patients with unilateral lateral epicondylalgia: a randomized controlled trial. JAMA 2013;309:461-9.

17 Smidt N, van der Windt DAWM, Assendelft WJJ, et al. Corticosteroid injections, physiotherapy, or a wait-and-see policy for lateral epicondylitis: a randomised controlled trial. Lancet 2002;359:657-62.

18 Bisset L, Beller E, Jull G, et al. Mobilisation with movement and exercise, corticosteroid injection, or wait and see for tennis elbow: randomised trial. BMJ 2006;333:939.

19 Karanasios S, Korakakis V, Whiteley R, et al. Exercise interventions in lateral elbow tendinopathy have better outcomes than passive interventions, but the effects are small: a systematic review and meta-analysis of 2123 subjects in 30 trials. Br J Sports Med 2021:55:477-85.

20 Hoogvliet P, Randsdorp MS, Dingemanse R, et al. Does effectiveness of exercise therapy and mobilisation techniques offer guidance for the treatment of lateral and medial epicondylitis? A systematic review. Br J Sports Med 2013;47:1112-9.

21 Coombes BK, Connelly L, Bisset L, et al. Economic evaluation favours physiotherapy but not corticosteroid injection as a firstline intervention for chronic lateral epicondylalgia: evidence from a randomised clinical trial. Br J Sports Med 2016;50:1400-5.

22 Dong W, Goost H, Lin X-B, et al. Injection therapies for lateral epicondylalgia: a systematic review and Bayesian network metaanalysis. Br J Sports Med 2016;50:900-8.

23 de Vos R-J, Windt J, Weir A. Strong evidence against platelet-rich plasma injections for chronic lateral epicondylar tendinopathy: a systematic review. Br J Sports Med 2014;48:952-6.

24 Yoon SY, Kim YW, Shin I-S, et al. Does the type of extracorporeal shock therapy influence treatment effectiveness in lateral epicondylitis? A systematic review and meta-analysis. Clin Orthop Relat Res 2020;478:2324-39.

25 Chang W-D, Lai P-T, Tsou Y-A. Analgesic effect of manual acupuncture and laser acupuncture for lateral epicondylalgia: a systematic review and meta-analysis. Am J Chin Med 2014;42:1301-14.

26 Watson T. Ultrasound in contemporary physiotherapy practice. Ultrasonics 2008;48:321-9.

27 Leighton R, Watson JT, Giannoudis P, et al. Healing of fracture nonunions treated with low-intensity pulsed ultrasound (LIPUS): a systematic review and meta-analysis. Injury 2017;48:1339-47.

28 Korstjens CM, Rutten S, Nolte PA, et al. Low-Intensity pulsed ultrasound increases blood vessel size during fracture healing in patients with a delayed-union of the osteotomized fibula. Histol Histopathol 2018;33:737-46.

29 Rutjes AW, Nüesch E, Sterchi R. Therapeutic ultrasound for osteoarthritis of the knee or hip. Cochrane Database Syst Rev 2010;1:CD003132.

30 Alfredo PP, Junior WS, Casarotto RA. Efficacy of continuous and pulsed therapeutic ultrasound combined with exercises for knee osteoarthritis: a randomized controlled trial. Clin Rehabil 2020;34:480-90.

31 Ebadi S, Henschke N, Forogh B, et al. Therapeutic ultrasound for chronic low back pain. Cochrane Database Syst Rev 2020;7:CD009169.

32 Altan L, Kasapoğlu Aksoy M, Kösegil Öztürk E. Efficacy of diclofenac \& thiocolchioside gel phonophoresis comparison with ultrasound therapy on acute low back pain; a prospective, double-blind, randomized clinical study. Ultrasonics 2019;91:201-5.

33 Lai WC, Iglesias BC, Mark BJ, et al. Low-Intensity pulsed ultrasound augments tendon, ligament, and Bone-Soft tissue healing in preclinical animal models: a systematic review. Arthroscopy 2021;37:2318-33.

34 Ebenbichler GR, Erdogmus CB, Resch KL, et al. Ultrasound therapy for calcific tendinitis of the shoulder. N Engl J Med 1999;340:1533-8.
35 Pieber K, Grim-Stieger M, Kainberger F, et al. Long-Term course of shoulders after ultrasound therapy for calcific tendinitis: results of the 10-year follow-up of a randomized controlled trial. Am J Phys Med Rehabil 2018;97:651-8.

36 Desmeules F, Boudreault J, Roy J-S, et al. The efficacy of therapeutic ultrasound for rotator cuff tendinopathy: a systematic review and meta-analysis. Phys Ther Sport 2015;16:276-84.

37 Chester R, Costa ML, Shepstone L, et al. Eccentric calf muscle training compared with therapeutic ultrasound for chronic Achilles tendon pain--a pilot study. Man Ther 2008;13:484-91.

38 Draper DO, Edvalson CG, Knight KL, et al. Temperature increases in the human Achilles tendon during ultrasound treatments with commercial ultrasound gel and full-thickness and half-thickness gel pads. J Athl Train 2010;45:333-7.

39 Stasinopoulos D, Stasinopoulos I. Comparison of effects of exercise programme, pulsed ultrasound and transverse friction in the treatment of chronic patellar tendinopathy. Clin Rehabil 2004; $18: 347-52$

40 Dingemanse R, Randsdorp M, Koes BW, et al. Evidence for the effectiveness of electrophysical modalities for treatment of medial and lateral epicondylitis: a systematic review. $\mathrm{Br} J$ Sports Med 2014;48:957-65.

41 Özmen T, Koparal SS, Karatas Özlem, et al. Comparison of the clinical and sonographic effects of ultrasound therapy, extracorporeal shock wave therapy, and Kinesio taping in lateral epicondylitis. Turk $J$ Med Sci 2021;51:76-83.

42 Dedes V, Tzirogiannis K, Polikandrioti M, et al. Comparison of radial extracorporeal shockwave therapy with ultrasound therapy in patients with lateral epicondylitis. J Med Ultrason 2020;47:319-25.

43 Yalvaç B, Mesci N, Geler Külcü D, et al. Comparison of ultrasound and extracorporeal shock wave therapy in lateral epicondylosis. Acta Orthop Traumatol Turc 2018;52:357-62.

44 Kubot A, Grzegorzewski A, Synder M, et al. Radial extracorporeal shockwave therapy and ultrasound therapy in the treatment of tennis elbow syndrome. Ortop Traumatol Rehabil 2017;19:415-26.

45 Lizis P. Analgesic effect of extracorporeal shock wave therapy versus ultrasound therapy in chronic tennis elbow. J Phys Ther Sci 2015;27:2563-7.

46 Coombes BK, Bisset L, Vicenzino B. Efficacy and safety of corticosteroid injections and other injections for management of tendinopathy: a systematic review of randomised controlled trials. Lancet 2010;376:1751-67.

47 Schwitzguebel AJ, Bogoev M, Nikolov V, et al. Tennis elbow, study protocol for a randomized clinical trial: needling with and without platelet-rich plasma after failure of up-to-date rehabilitation. $J$ Orthop Surg Res 2020;15:462.

48 Keijsers R, Kuijer PPFM, Koenraadt KLM, et al. Effectiveness of standardized ultrasound guided percutaneous treatment of lateral epicondylitis with application of autologous blood, dextrose or perforation only on pain: a study protocol for a multi-center, blinded, randomized controlled trial with a 1 year follow up. BMC Musculoskelet Disord 2019;20:351.

49 Lungu E, Grondin P, Tétreault P, et al. Ultrasound-Guided tendon fenestration versus open-release surgery for the treatment of chronic lateral epicondylosis of the elbow: protocol for a prospective, randomised, single blinded study. BMJ Open 2018;8:e021373.

50 Chan A-W, Tetzlaff JM, Gøtzsche PC, et al. Spirit 2013 explanation and elaboration: guidance for protocols of clinical trials. BMJ 2013;346:e7586.

51 Usuelli FG, Di Silvestri CA, D'Ambrosi R, et al. Return to sport activities after medial displacement calcaneal osteotomy and flexor digitorum longus transfer. Knee Surg Sports Traumatol Arthrosc 2018;26:892-6

52 Coombes BK, Bisset L, Connelly LB, et al. Optimising corticosteroid injection for lateral epicondylalgia with the addition of physiotherapy: a protocol for a randomised control trial with placebo comparison. BMC Musculoskelet Disord 2009;10:76.

53 Vicenzino B. Lateral epicondylalgia: a musculoskeletal physiotherapy perspective. Man Ther 2003;8:66-79.

54 Rompe JD, Overend TJ, MacDermid JC. Validation of the Patient-rated tennis elbow evaluation questionnaire. $J$ Hand Ther 2007;20:3-11.

55 Giray E, Karali-Bingul D, Akyuz G. The effectiveness of Kinesiotaping, sham taping or exercises only in lateral epicondylitis treatment: a randomized controlled study. Pm R 2019;11:681-93.

56 Poltawski L, Watson T. Measuring clinically important change with the Patient-rated tennis elbow evaluation. Hand Therapy 2011;16:52-7.

57 Leung HB, Yen CH, Tse PYT. Reliability of Hong Kong Chinese version of the Patient-rated forearm evaluation questionnaire for lateral epicondylitis. Hong Kong Med J 2004;10:172-7. 
58 Jensen MP, Chen C, Brugger AM. Interpretation of visual analog scale ratings and change scores: a reanalysis of two clinical trials of postoperative pain. J Pain 2003;4:407-14.

59 Beaton DE, Wright JG, Katz JN, et al. Development of the QuickDASH: comparison of three item-reduction approaches. J Bone Joint Surg Am 2005;87:1038-46.

60 Lerner D, Amick BC, Rogers WH, et al. The work limitations questionnaire. Med Care 2001;39:72-85.

61 EuroQol Group. EuroQol--a new facility for the measurement of health-related quality of life. Health Policy 1990;16:199-208.

62 Zigmond AS, Snaith RP. The hospital anxiety and depression scale. Acta Psychiatr Scand 1983;67:361-70.

63 Mahomed N, Gandhi R, Daltroy L, et al. The self-administered patient satisfaction scale for primary hip and knee arthroplasty. Arthritis 2011:2011:1-6.

64 Stratford PW, Levy DR, Gauldie S. Extensor carpi radialis tendonitis: a validation of selected outcome measures. Physiotherapy Canada 1987;39:250-5.

65 Shin K-M, Kim J-H, Lee S, et al. Acupuncture for lateral epicondylitis (tennis elbow): study protocol for a randomized, practitioner-assessor blinded, controlled pilot clinical trial. Trials 2013;14:174

66 Cao S, Zhou R, Zhou H, et al. Reliability and validity of simplified Chinese version of quick disabilities of the arm, shoulder, and hand (QuickDASH) questionnaire: cross-cultural adaptation and validation. Clin Rheumatol 2019;38:3281-7.

67 Franchignoni F, Vercelli S, Giordano A, et al. Minimal clinically important difference of the disabilities of the arm, shoulder and hand outcome measure (DASH) and its shortened version (QuickDASH). $J$ Orthop Sports Phys Ther 2014;44:30-9.
68 Smidt N, van der Windt DA, Assendelft WJ, et al. Interobserver reproducibility of the assessment of severity of complaints, grip strength, and pressure pain threshold in patients with lateral epicondylitis. Arch Phys Med Rehabil 2002;83:1145-50.

69 Roy J-S, MacDermid JC, Amick BC, et al. Validity and responsiveness of presenteeism scales in chronic work-related upper-extremity disorders. Phys Ther 2011;91:254-66.

70 Wu C, Gong Y, Wu J, et al. Chinese version of the EQ-5D preference weights: applicability in a Chinese general population. PLoS One 2016;11:e0164334.

71 Sun S, Chen J, Johannesson M, et al. Population health status in China: EQ-5D results, by age, sex and socio-economic status, from the National health services survey 2008. Qual Life Res 2011;20:309-20.

72 Alizadehkhaiyat O, Fisher AC, Kemp GJ, et al. Pain, functional disability, and psychologic status in tennis elbow. Clin J Pain 2007;23:482-9.

73 Pallant JF, Bailey CM. Assessment of the structure of the hospital anxiety and depression scale in musculoskeletal patients. Health Qual Life Outcomes 2005;3:82.

74 Razmjou H, Holtby R. Impact of rotator cuff tendon reparability on patient satisfaction. JSES Open Access 2017;1:5-9.

75 Rabago D, Lee KS, Ryan M, et al. Hypertonic dextrose and morrhuate sodium injections (prolotherapy) for lateral epicondylosis (tennis elbow): results of a single-blind, pilot-level, randomized controlled trial. Am J Phys Med Rehabil 2013;92:587-96.

76 Sedgwick P. Intention to treat analysis versus per protocol analysis of trial data. BMJ 2015;350:h681.

77 Detry MA, Ma Y. Analyzing repeated measurements using mixed models. JAMA 2016;315:407-8. 